\title{
Simple Organic Light-Emitting Devices with a Charge Carrier Modulation Layer Towards Lower Driving Voltage and Higher Electroluminescent Efficiency
}

\author{
Qin Xue, ${ }^{a}$ Yang Li, ${ }^{a}$ Xianchun Zhang, ${ }^{a}$ Yongxin Bian, ${ }^{a}$ Heng Hu, ${ }^{a}$ and Guohua Xie ${ }^{*, b}$ \\ ${ }^{a}$ Department of Physical Science and Technology, Central China Normal University, Wuhan, Hubei 430079, China \\ ${ }^{b}$ College of Chemistry and Molecular Sciences, Wuhan University, Wuhan, Hubei 430072, China
}

\begin{abstract}
We reported on simple but novel organic light-emitting devices with a thin electron transport layer inserted between a hole-injecting layer and a hole-transporting layer. Unexpectedly, it was observed that charge carriers could easily tunnel through the thin but high barrier and contribute to lower driving voltage and higher electroluminescent efficiency. When fixing the modulation layer for charge carrier tunneling at a thickness of $2.5 \mathrm{~nm}$, we could achieve over $80 \%$ improvement in the power efficiency.
\end{abstract}

Keywords organic light-emitting devices, tunnels, barrier, trapping, reverse field

\section{Introduction}

The evolution of organic light-emitting device (OLED) technology during the past three decades has attracted intensive attention from not only academics but also industry. Attributed to the fast development of organic luminescent materials, from fluorescence, ${ }^{[1]}$ phosphorescence, ${ }^{[2-4]}$ to thermally activated delayed fluorescence, ${ }^{[5-9]}$ OLEDs become definitely one of the most promising candidates for the next generation technology of displays and lighting. ${ }^{[10-16]}$ However, the evolution of device engineering and physics is left behind the fast development of chemistry and materials of organic semiconductors. Inheriting from physics of inorganic semiconductors, organic semiconducting devices can be easily modified and engineered. Unlike inorganic semiconductors, organic semiconductors possess considerably low charge carrier mobility. Therefore, OLEDs exhibited usually higher driving voltages, especially at high brightness, compared to the LED counterparts. Due to low mobility materials used in OLEDs, the devices were usually kept as thin and simple as possible. To improve charge injection and transport in OLEDs, high mobility materials, energy alignment scheme, and electrical doping strategies have been introduced. ${ }^{[4,12,13]}$ Herein, we proposed a simple approach to engineer charge injection in OLEDs without complicating device fabrication. Based on a tunneling process, i.e., inserting a thin layer of high mobility electron transport material with low level of highest occupied molecular orbital (HOMO) between a hole-injecting layer (HIL) and a hole-transporting layer (HTL), in principle, holes can be feasibly injected into an emitting layer (EML), vice versa for electrons, and thus reduce simultaneously the driving voltage and improve the luminous efficacy.

\section{Experimental}

Pre-patterned indium-tin-oxide (ITO) substrates were cleaned consecutively in acetone and ethanol for $10 \mathrm{~min}$, respectively. After dried out with nitrogen, the substrates were loaded into a UV-ozone generator for a surface treatment for $10 \mathrm{~min}$. Then the ITO substrates were loaded into a thermal evaporator which was pumped down to $\sim 2 \times 10^{-4} \mathrm{~Pa}$ before evaporation of organic materials. All materials were deposited layer by layer with a rate of approximately $0.1 \mathrm{~nm} / \mathrm{s}$ for organic materials, $0.01 \mathrm{~nm} / \mathrm{s}$ for LiF, and finally $1 \mathrm{~nm} / \mathrm{s}$ for Al, respectively. All organic materials were purchased from Lumtec (Taiwan, China) and used as received without further purification. For characterization of electroluminescent performance, a spectrometer (Photo Research 650) was combined with a source meter unit (Keithley 2400) controlled by a customized measurement program.

\section{Results and Discussion}

A simple non-doped architecture was constructed as follows: ITO/m-MTDATA $((45-x) \mathrm{nm}) /$ BPhen $(x$ $\mathrm{nm}) / \mathrm{NPB}(5 \mathrm{~nm}) / \mathrm{Alq}_{3}(55 \mathrm{~nm}) / \mathrm{LiF}(1 \mathrm{~nm}) / \mathrm{Al}(100 \mathrm{~nm})$, where $x=0,2.5$ and 5 for devices $\mathrm{A}, \mathrm{B}$ and $\mathrm{C}$, respec-

\footnotetext{
* E-mail:xgh-008@163.com.

Received October 29, 2016; accepted November 4, 2016.
} 
tively. Here, $m$-MTDATA denotes 4,4',4"tris(3-methylphenylphenylamino)triphenylamine, which is a typical hole-injecting layer used for OLEDs. BPhen is 4,7-diphenyl-1,10-phenanthroline, which is commonly used as an electron transport layer. However, it is used in this work as a charge carrier tunneling medium due to its deep highest occupied molecular orbital (HOMO) down to $6.4 \mathrm{eV}$ and thus high barrier for holes. NPB denotes $N, N^{\prime}$-diphenyl- $N, N^{\prime}$-bis(1,1'-bi-phenyl)4,4'-diamine, which serves as a hole-transporting layer. ${ }^{[17-20]} \mathrm{Alq}_{3}$ is short for tris-(8-hydroxyquinoline) aluminum, which is a fluorescent light-emitting material possessing a decent electron mobility. Figure 1 shows molecular structures used in this investigation and the device architecture.

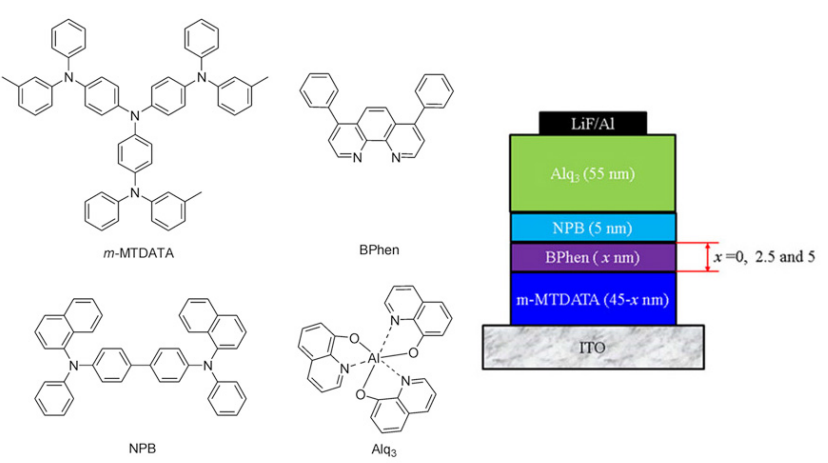

Figure 1 Molecular structures of organic materials and the structure of devices.

Figure 2 presents that the lowest unoccupied molecular orbital (LUMO) and HOMO of BPhen are much lower than those of $m$-MTDATA and NPB, respectively. At the interface of $m$-MTDATA and BPhen, the energy barrier for hole-injection is up to $1.3 \mathrm{eV}$. However, there is only $0.3 \mathrm{eV}$ for hole-injection barrier from $m$-MTDATA to NPB without a thin layer of BPhen. In terms of electron transport, the electrons can be easily trapped at the well-like sites on BPhen due to the low LUMO level of BPhen. However, the electrons will be mainly blocked and accumulated at the interface of NPB and $\mathrm{Alq}_{3}$ as the energy offset is about $0.7 \mathrm{eV}$, which is higher than that between LUMOs of $m$-MTDATA and NPB $(0.5 \mathrm{eV})$. At the presence of BPhen, the electrons tend to be trapped and accumulated at the interface of $m$-MTDATA and BPhen due to the large energy offset up to $1.1 \mathrm{eV}$. Electrons/holes can be injected via tunneling for either depletion or excess injection of opposite charge carriers. ${ }^{[21-23]}$ It is reasonable that we can engineer the charge carrier injection and redistribution and thus improve the electroluminescent (EL) performances by introducing a thin charge carrier modulation layer of BPhen with high electron mobility and large energy level offset.

Based on analysis of the proposed architecture, we compared the EL performances of devices with three

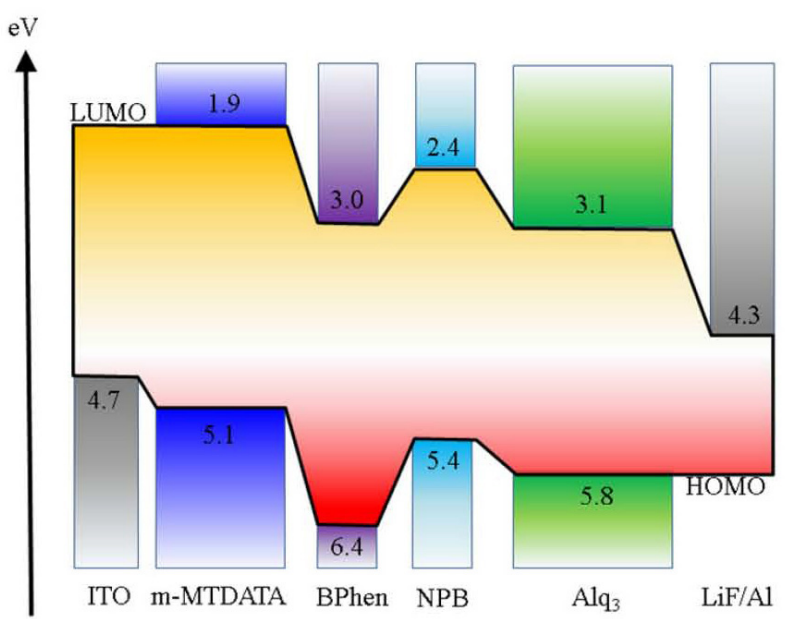

Figure 2 Schematic diagram of energy levels of devices.
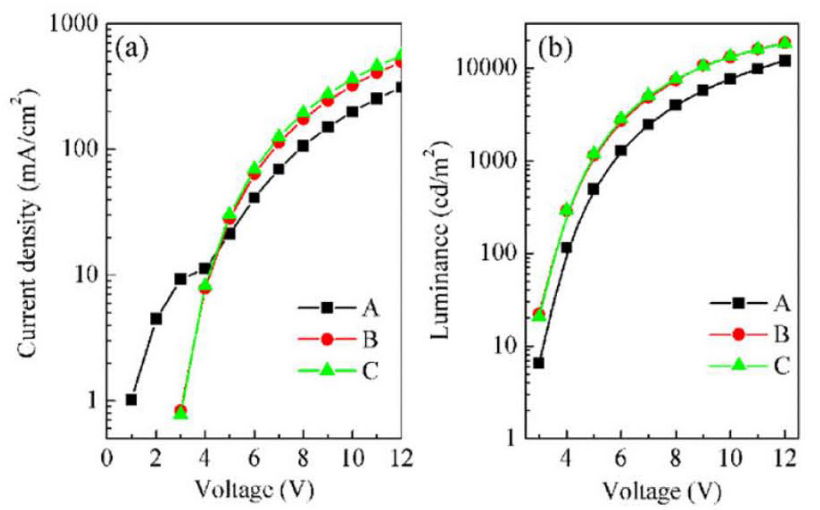

Figure 3 Current density (a) and luminance (b) vs. driving voltage of devices.

different thicknesses of BPhen, i.e., 0, 2.5 and $5 \mathrm{~nm}$. The current density-voltage characteristics are shown in Figure 3a. Under low bias, e.g., 4V, the device without BPhen rendered higher current density. However, the current densities of devices $\mathrm{B}$ and $\mathrm{C}$ with 2.5 and 5 $\mathrm{nm}$-thick PBhen increased steeply under higher biased voltage, respectively. The luminance-voltage curves of devices $\mathrm{B}$ and $\mathrm{C}$ were higher than that of device A (Figure $3 b$ ).

Figures $4 a-4 c$ displayed current efficiency, power efficiency and external quantum efficiency for three devices, respectively. Without the BPhen layer, the efficiencies are sufficiently low at small current density $(<$ $10 \mathrm{~mA} / \mathrm{cm}^{2}$ ), which can be ascribed to the high leakage current (Figure 3a). In contrast, the efficiencies are nearly independent on the current density by introducing a thin layer of BPhen between HIL and HTL. The maximum current efficiencies of devices $\mathrm{A}, \mathrm{B}$ and $\mathrm{C}$ are $3.9,4.4$ and $4.0 \mathrm{~cd} / \mathrm{A}$. The power efficiencies are 1.6, 2.9 and $2.8 \mathrm{~lm} / \mathrm{W}$. Compared with the maximum external quantum efficiency of device A $(1.3 \%)$, an improvement of $13 \%$ and $3 \%$ can be achieved for devices $\mathrm{B}$ and $\mathrm{C}$, respectively. 

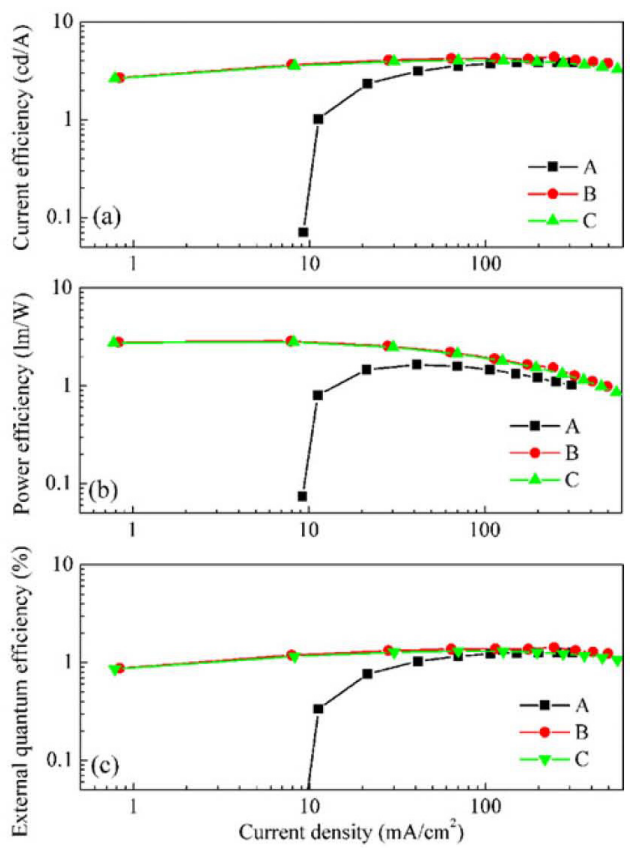

Figure 4 Current efficiency (a), power efficiency (b), and external quantum efficiency (c) $v s$. current density of devices.

It was reported that an exciplex emission can be easily observed due to a small energy gap $(2.1 \mathrm{eV})$ between HOMO of $m$-MTDATA and LUMO of BPhen. ${ }^{[24-26]}$ In this regards, we compared the normalized EL spectra of the devices with and without BPhen in Figure 5. Undoubtedly, we found no difference of the EL spectra as they completely overlapped with each other. Therefore, the recombination zone is supposed to be well confined at the vicinity of the interface between NPB and $\mathrm{Alq}_{3}$, which was a typical feature of $\mathrm{NBP} / \mathrm{Alq}_{3}$-like structure. ${ }^{[27]}$

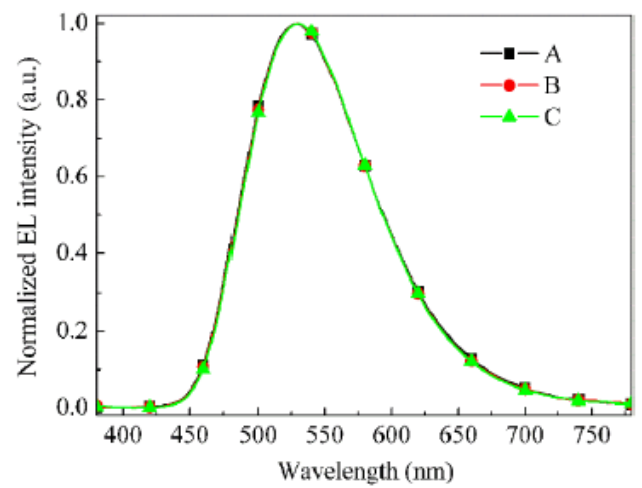

Figure 5 Normalized EL spectra of devices.

To further explore the property of the charge carrier injection and transport with an additional layer of BPhen between HIL and HTL, we constructed a so-called "hole-only" device for probing the hole current with and without BPhen. Figure 6a showed that the "hole-only" device replaced $\mathrm{Alq}_{3}$ with $m$-MTDATA covered with a layer of $\mathrm{Al}$ for electrical contact. It is supposed that the device is a hole-dominant one and no electron current is injected. Figure $6 \mathrm{~b}$ presented that the current is space charge limited when applying a positive bias at the ITO side and a negative bias at the Al side. The current density was reduced when the thickness of BPhen increased. When applying the positive bias at the Al side and the negative bias at the ITO side, the current density was much lower than that at the opposite bias above $2 \mathrm{~V}$ and it was almost independent on the thickness of BPhen. Interestingly, in this case, a slightly improved current density could be observed when the thickness of BPhen was kept at $2.5 \mathrm{~nm}$. Therefore, we assume that the holes can tunnel through easily BPhen once the thickness is thin enough (e.g., $<5 \mathrm{~nm}$ ) and thus induce the electron injection under internal electric field enhancement.
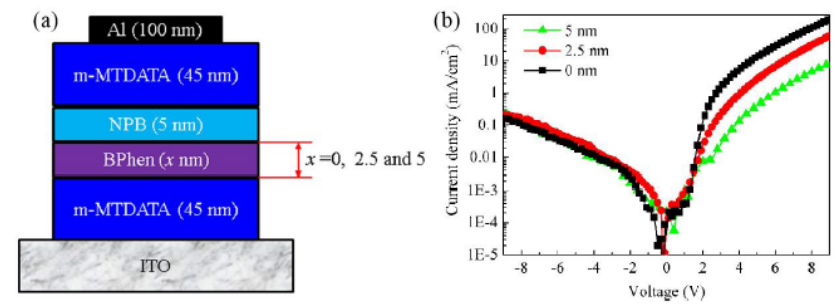

Figure 6 (a) The structure of the "hole-only" devices with different thicknesses of BPhen. (b) Current density vs. voltages of the "hole-only" devices.

\section{Conclusions}

In summary, we constructed a novel OLED with simple non-doping strategy to investigate the influence of a thin tunnel layer on the performances of the device. As the tunnel layer is thin enough, the exciplex emission between the donor-like and acceptor-like transporting materials can be refrained as the recombination zone is well separated. The enhanced EL performances could be ascribed to trap-assisted tunneling and reverse electric field enhancement in a working OLED. In this embodiment, we realized simultaneously reduction of operating voltage at high brightness and enhancement of power efficiency. Compared with the control device without BPhen, the maximum power efficiencies are improved by $81 \%$ and $75 \%$ for the devices with 2.5 and $5 \mathrm{~nm}$-thick BPhen, respectively. This work provides a simple and effective method to engineer the charge carrier in the OLEDs, which can be applied to other devices.

\section{Acknowledgements}

We gratefully acknowledged financial supports from the National Natural Science Foundation of China (No. 61405069) and self-determined research funds of Central China Normal University from the colleges' basic research and operation of MOE (No. CCNU15A02038). 


\section{References}

[1] Tang, C. W.; VanSlyke, S. A. Appl. Phys. Lett. 1987, 51, 913

[2] Baldo, M. A.; O'Brien, D. F.; You, Y.; Shoustikov, A.; Sibley, S.; Thompson, M. E.; Forrest, S. R. Nature 1998, 395, 151.

[3] Sun, Y.; Giebink, N. C.; Kanno, H.; Ma, B.; Thompson, M. E.; Forrest, S. R. Nature 2006, 440, 908.

[4] Reineke, S.; Lindner, F.; Schwartz, G.; Seidler, N.; Walzer, K.; Lüssem, B.; Leo, K. Nature 2009, 459, 234.

[5] Uoyama, H.; Goushi, K.; Shizu, K.; Nomura, H.; Adachi, C. Nature 2012, 492, 234.

[6] Lee, S. Y.; Yasuda, T.; Nomura, H.; Adachi, C. Appl. Phys. Lett. 2012, 101, 093306.

[7] Zhang, Q.; Li, J.; Shizu, K.; Huang, S.; Hirata, S.; Miyazaki, H.; Adachi, C. J. Am. Chem. Soc. 2012, 134, 14706.

[8] Lee, S. Y.; Yasuda, T.; Yang, Y. S.; Zhang, Q.; Adachi, C. Angew. Chem., Int. Ed. 2014, 126, 6520.

[9] Hirata, S.; Sakai, Y.; Masui, K.; Tanaka, H.; Lee, S. Y.; Nomura, H.; Nakamura, N.; Yasumatsu, M.; Nakanotani, H.; Zhang, Q.; Shizu, K.; Miyazaki, H.; Adachi, C. Nat. Mater. 2015, 14, 330.

[10] www.universaldisplay.com

[11] www.cdt.co.uk

[12] Xie, G.; Xue, Q.; Chen, P.; Tao, C.; Zhao, C.; Lu, J.; Gong, Z.; Zhang, T.; Huang, R.; Du, H.; Xie, W.; Hou, J.; Zhao, Y.; Liu, S. Org. Electron. 2010, 11, 407.

[13] Xie, G.; Zhang, Z.; Xue, Q.; Zhang, S.; Zhao, L.; Luo, Y.; Chen, P.; Quan, B.; Zhao, Y.; Liu, S. Org. Electron. 2010, 112055.
[14] Han, C.; Xie, G.; Xu, H.; Zhang, Z.; Xie, L.; Zhao, Y.; Liu, S.; Huang, W. Adv. Mater. 2011, 23, 2491.

[15] Xie, G.; Zhang, Z.; Xue, Q.; Zhang, S.; Luo, Y.; Zhao, L.; Chen, P.; Quan, B.; Zhao, Y.; Liu, S. J. Phys. Chem. C 2011, 115, 264.

[16] Fung, M.; Li, Y.; Liao, L. Adv. Mater. 2016, DOI: 10.1002/adma.201601737.

[17] Sun, M.; Zhu, W.; Zhang, Z.; Ou, C.; Xie, L.; Yang, Y.; Qian, Y.; Zhao, Y.; Huang, W. J. Mater. Chem. C 2015, 3, 94.

[18] Cao, H.; Sun, H.; Yin, Y.; Wen, X.; Shan, G.; Su, Z.; Zhong, R.; Xie, W.; Li, P.; Zhu, D. J. Mater. Chem. C 2014, 2, 2150.

[19] Cao, H.; Shan, G.; Wen, X.; Sun, H.; Su, Z.; Zhong, R.; Xie, W.; Li, P.; Zhu, D. J. Mater. Chem. C 2013, 1, 7371.

[20] Xie, L.; Zhu, R.; Qian, Y.; Liu, R.; Chen, S.; Lin, J.; Huang, W. J. Phys. Chem. Lett. 2010, 1, 272.

[21] Lu, M.; Bruyn, P.; Nicolai, H. T.; Wetzelaer, G. A. H.; Blom, P. W. M. Org. Electron. 2012, 13, 1693.

[22] Teramoto, A.; Kobayashi, K.; Ohno, Y.; Shigetomi, A. IEEE T. Electron Dev. 2001, 48, 868.

[23] Xie, G.; Fehse, K.; Leo, K.; Gather, M. C. Org. Electron. 2013, 14. 2331.

[24] Goushi, K.; Yoshida, K.; Sata, K.; Adachi, C. Nature Photon. 2012, $6,253$.

[25] Graves, D.; Jankus, V.; Dias, F. B.; Monkman, A. Adv. Funct. Mater. 2014, 24, 2343.

[26] Zhu, L.; Xu, K.; Wang, Y.; Chen, J.; Ma, D. Front. Optoelectron. 2015, 8, 439.

[27] Lin, T.; Hsiao, C.; Lee, J. Proc, SPIE 2005, 5937, 59371Q. 